\title{
Impact of molecular weight and serotype on botulinum neurotoxin oral toxicity and stability in simulated gastrointestinal tract conditions
}

\section{J. Adam Crawford}

Applied Biology and Aerosol Technology,

Battelle Memorial Institute,

505 King Ave., Columbus Ohio, 43201-4696, USA

Fax: 614-458-4407

E-mail: CrawfordJA@Battelle.org

\section{Jennifer Kronenberg}

Atlanta Analytical Services,

Battelle Memorial Institute, 1600 Clifton Rd NE, M/S G-18, Atlanta, GA, 30329-4018, USA

E-mail: JKronenberg@cdc.gov

\section{Jennifer Beare}

Applied Biology and Aerosol Technology,

Battelle Memorial Institute,

505 King Ave. Columbus Ohio, 43201-4696, USA

Fax: 614-458-4537

E-mail: BeareJ@Battelle.org

\section{Rebekah Starner}

Battelle Biomedical Research Center, Battelle Memorial Institute, 1425 Plain City - Georgesville Route 142, West Jefferson, OH 43162-9647, USA

Fax: 614-458-5240

E-mail: Starner@Battelle.org

\section{Angelo Scorpio}

National Biodefense Analysis and Countermeasures Center (NBACC),

8300 Research Plaza, Fort Detrick, MD 21702-9211, USA

E-mail: Angelo.Scorpio@nbacc.dhs.gov 


\title{
Sherry Blight*
}

Applied Biology and Aerosol Technology, Battelle Memorial Institute, 505 King Ave. Columbus Ohio, 43201-4696, USA

Fax: 614-458-7800

E-mail: BlightS@Battelle.org

*Corresponding author

\begin{abstract}
The botulinum neurotoxins (BoNTs) are among the most lethal substances known to humankind. The work described herein examined the oral toxicity of the BoNTs and compared oral toxicity to in vitro stability in simulated gastrointestinal tract conditions. Our results demonstrate that a majority of the BoNTs tested were less stable in simulated intestinal fluid (SIF) as compared to simulated gastric fluid (SGF), and that oral toxicity correlated with the molecular weight of the BoNT complex. These results suggest that stability in the intestinal tract plays a critical role in the oral toxicity of the BoNTs.
\end{abstract}

Keywords: botulinum; neurotoxin; botulinum neurotoxin; BoNT; simulated gastric fluid; SGF; simulated intestinal fluid; SIF; molecular weight; MW; oral toxicity; serotype; stability; gastrointestinal tract.

Reference to this paper should be made as follows: Crawford, J.A., Kronenberg, J., Beare, J., Starner, R., Scorpio, A. and Blight, S. (2012) 'Impact of molecular weight and serotype on botulinum neurotoxin oral toxicity and stability in simulated gastrointestinal tract conditions', The Botulinum J., Vol. 2, No. 2, pp.109-118.

Biographical notes: J. Adam Crawford holds a $\mathrm{PhD}$ in Microbiology from the University of Michigan and conducted postdoctoral research at the Center for Vaccine Development at the University of Maryland. He has over 18 years of experience in vaccine research, bacterial pathogenesis, microbiology, biochemistry, and molecular biology.

Jennifer Kronenberg received her Bachelor's in Biology from Bowling Green State University. She currently serves as the In Vitro Diagnostic (IVD) Manufacturing Manager at CDC Influenza Division where the current focus is on the design and development of multiple rRT-PCR influenza diagnostic assays that are used for surveillance and diagnosis of influenza in domestic public health laboratories as well as to detect the presence of novel or emerging influenza strains.

Jennifer Beare is a Senior Technician at Battelle. She received her BS in Microbiology from The Ohio State University and has four years of laboratory experience in the areas of microbiology, molecular biology and immunobiology.

Rebekah Starner is a Senior Research Scientist at Battelle where she has been employed for over 26 years. Her present duties are to design and conduct FDA regulated studies utilising the mouse neutralisation assay and potency assay used to quantify the anti-botulinum antibodies to specific botulinum serotypes (A-G) in various matrices such as serum, plasma, concentrated antibodies of various species such as human, guinea pig, mouse, sheep, goat and horse. She was the Study Director on the task that supported the licensure of the botulism immune globulin product Baby BIG ${ }^{\mathbb{R}}$ used for the treatment of infant botulism. 


\begin{abstract}
Angelo Scorpio is a Principal Investigator at the National Biodefense Analysis and Countermeasures Center, Fort Detrick, MD. His research interests include microbial pathogenesis, with emphasis on virulence mechanisms of Bacillus anthracis. His current research efforts are focused on characterisation of biological threat agents to inform biodefense planning and facilitate the development of medical countermeasures.
\end{abstract}

Sherry Blight is a Research Scientist at Battelle Memorial Institute. She received her $\mathrm{PhD}$ in Microbiology from The Ohio State University, and has over 15 years of academic and government laboratory experience in microbiology, biochemistry, physiology, and metabolic engineering.

\title{
1 Introduction
}

The botulinum neurotoxins (BoNTs) are a group of structurally similar proteins produced by Clostridium botulinum and related clostridial species (including C. baratii and C. butyricum). The BoNTs cause the potentially lethal disease botulism in humans, which is historically associated with ingestion of contaminated food. Seven immunologically distinct BoNT serotypes are currently known (serotypes A-G). Although structurally similar, only a subset of the BoNT serotypes (serotypes A, B, E, and less frequently serotype $\mathrm{F}$ ) is commonly associated with human disease.

Following ingestion, the BoNTs are absorbed through the mucous membrane of the stomach (Maksymowych et al., 1999) or the epithelial barrier of the small intestine (Bonventre, 1979); thus, the neurotoxin must survive the harsh environment of the gastrointestinal tract for severe intoxication or death to occur. Previous studies demonstrated that high molecular weight (MW) forms of the BoNTs displayed increased oral toxicity (Ohishi et al., 1977; Sakaguchi and Sakaguchi, 1974; Sugiyama et al., 1974), and enhanced in vitro stability under acidic conditions or in the presence of gastric and intestinal fluids (Sugii et al., 1977a, 1977b). Higher MW forms of the BoNTs contain various non-toxic accessory proteins including hemagglutinin (HA) proteins and proteins that lack HA activity (non-toxic non-HA; NTNHs) (Inoue et al., 1996; Jin et al., 2009; Sakaguchi and Ohishi, 1984), and it is thought that these accessory proteins protect the neurotoxin polypeptide against stomach acidity and degradative enzymes in the gastrointestinal tract (Sakaguchi and Ohishi, 1984), and may also allow binding to the microvilli of epithelial cells of the small intestine (Fujinaga et al., 1997). However, it has been demonstrated that the neurotoxin alone, minus the accessory proteins, can still undergo transcytosis in both the stomach and the intestine (Maksymowych et al., 1999; Maksymowych and Simpson, 2004). The interaction of the BoNTs with the epithelial barrier is reviewed by Fujinaga (2010).

It has been proposed that the oral administration of botulinum vaccines may be an effective strategy for protecting against BoNT intoxication (Kiyatkin et al., 1997). Thus, there is a need to better understand relative oral toxicities across multiple BoNT serotypes, the impact of MW on toxicity, and the impact of MW on in vitro stability. To address these needs, and to expand upon previous studies that examined the relationship between oral toxicity, MW, and in vitro stability (Ohishi et al., 1977; Sakaguchi and Sakaguchi, 1974; Sugii et al., 1977a, 1977b; Sugiyama et al., 1974), a systematic analysis was performed to compare the oral toxicity of multiple BoNT serotypes in mice and the 
impact of simulated gastric fluid (SGF; containing pepsin) and simulated intestinal fluid (SIF; containing pancreatin) on in vitro stability.

The specific BoNT serotypes selected for evaluation were A, B, C, E, and F. The crude (concentrated culture lysates), progenitor (purified protein complexes made up of BoNT plus various accessory proteins), and derivative (purified neurotoxin without accessory proteins) forms of each serotype were evaluated. Serotypes A, B, E, and F were assessed because this group of serotypes is associated with human disease. Serotype C, associated with botulism in animals, was included to compare its properties with the serotypes that cause human disease. Results of this work demonstrated that, in general, BoNT MW correlated with oral toxicity and impacted stability in both SGF and SIF.

\section{Materials and methods}

\subsection{BoNT preparations}

Crude, progenitor, and derivative BoNT preparations of serotype A, serotype B, serotype $\mathrm{C}$, serotype $\mathrm{E}$, and serotype $\mathrm{F}$ were purchased from Metabiologics, Inc. (Madison, WI; $>90 \%$ purity for progenitor and derivative preparations). The potency of the BoNT samples ranged from $6.0 \times 10^{6}$ to $2.2 \times 10^{8}$ mouse intraperitoneal median lethal dose per millilitre $\left(\mathrm{MIPLD}_{50} / \mathrm{mL}\right)$ at a final protein concentration of $1 \mathrm{mg} / \mathrm{mL}$. The identity of each preparation was confirmed by a mouse neutralisation assay using serotype specific antibodies.

\subsection{SGF and SIF preparation}

SGF (Fisher Scientific, Pittsburgh, PA) consisted of $0.2 \%(\mathrm{w} / \mathrm{v})$ sodium chloride in $0.7 \%$ $(\mathrm{v} / \mathrm{v})$ hydrochloric acid. Pepsin (Fisher Scientific) was added to the SGF to a final concentration of 3.2 grams per litre $(\mathrm{g} / \mathrm{L})$, per the United States Pharmacopeia - National Formulary (USP-NF) specifications. The $\mathrm{pH}$ of the SGF/pepsin solution was 1.2.

SIF (Fisher Scientific) consisted of $0.05 \mathrm{M} \mathrm{KH}_{2} \mathrm{PO}_{4}$ with $1 \mathrm{~N} \mathrm{NaOH}$ added so that the final $\mathrm{pH}$ was 7.5 (Chang and Gupta, 1996; Mao et al., 2006). Pancreatin (a mixture of several digestive enzymes including amylase, lipase and trypsin; Fisher Scientific) was added to the SIF to a final concentration of $1 \mathrm{~g} / \mathrm{L}$, per USP-NF formulation. The $\mathrm{pH}$ of the SIF/pancreatin solution was 7.4.

\subsection{Trypsin treatment of serotype $E$}

Prior to the intragastric (IG) challenge studies and the SGF/pepsin and SIF/pancreatin exposure studies, the serotype $\mathrm{E}$ preparations were treated with trypsin to activate the toxins. The other toxin preparations (serotypes A, B, C, and F) were from proteolytic strains and thus did not require trypsin activation. L-1-tosylamido-2-phenylethyl chloromethyl ketone (TPCK) trypsin beads (Pierce Biotechnology, Rockford, IL) were used to activate the serotype E preparations for the SGF/pepsin and SIF/pancreatin exposure studies, according to the manufacturer's recommendations. Specifically, 4.3 $p$-toluenesulfonyl-L-arginine methyl ester (TAME) units were added to one $\mathrm{mg}$ of the serotype $\mathrm{E}$ samples and the mixture incubated at $37^{\circ} \mathrm{C}$ for $30 \mathrm{~min}$. After incubation, the reactions were centrifuged at $12,000 \mathrm{rpm}$ for 5 minutes to pellet the beads, and the 
supernatants were removed and stored at $2^{\circ} \mathrm{C}$ to $8^{\circ} \mathrm{C}$ until used. For the IG challenge studies, the serotype $\mathrm{E}$ samples were adjusted to a protein concentration of $0.5 \mathrm{mg} / \mathrm{mL}$ and were then treated with TPCK-trypsin (Sigma-Aldrich, St. Louis, MO) at a final concentration of $100 \mu \mathrm{g} / \mathrm{mL}$ for 1 hour at $37^{\circ} \mathrm{C}$.

\subsection{SGF/Pepsin and SIF/pancreatin exposure assays}

For each serotype and neurotoxin form, 10,000 MIPLD $_{50}$ units were exposed to SGF/pepsin, SIF/pancreatin, or phosphate buffered saline (PBS; unexposed controls) for $30 \mathrm{~min}$ at $35^{\circ} \mathrm{C}$ in a $1 \mathrm{~mL}$ volume. Following incubation, $9 \mathrm{~mL}$ of chilled $0.2 \%$ gelatin phosphate buffer were added to each sample, followed by rapid freezing using ethanol/dry ice, and stored at $-70^{\circ} \mathrm{C}$ until use in the mouse potency assay (MPA).

\subsection{Mouse challenge studies}

\subsubsection{Mouse potency assay}

Five male CD-1 (ICR) mice (Mus musculus, Charles River Laboratories, Boston, MA) per group were challenged with one of six dose levels of each toxin preparation, for a total of 30 mice for each toxin preparation. BoNT samples were diluted to target MIPLD $_{50} / \mathrm{mL}$ concentrations in $0.2 \%$ gelatin phosphate buffer $(0.2 \%$ gelatin, $28.2 \mathrm{mM}$ sodium phosphate dibasic, $\mathrm{pH}$ 6.2). A $500 \mu \mathrm{L}$ dose of each preparation was administered to mice by intraperitoneal (IP) injection using a $27 \mathrm{G} \times 1 / 2$ inch needle, and the mice were monitored for lethality for $96 \pm 2 \mathrm{~h}$ after inoculation. At the end of the observation period, mortality was tabulated and all surviving mice were euthanised. The $\operatorname{MIPLD}_{50} / \mathrm{mL}$ was calculated using probit analysis based on the number of mouse deaths.

\subsubsection{IG challenge assay}

Eight groups of CD-1 (ICR) male mice (eight mice per group) were challenged with one of eight dose levels of each neurotoxin preparation, for a total of 64 mice for each toxin preparation. BoNT samples were diluted to target $\mathrm{MIPLD}_{50} / \mathrm{mL}$ concentrations in PBS containing $0.2 \%(\mathrm{w} / \mathrm{v})$ gelatin. A dose of $200 \mu \mathrm{L}$ was administered by oral gavage using a $20 \mathrm{G} \times 1 \frac{1}{2}$ inch disposable orogastric intubation needle, and the mice were monitored for up to 14 days post challenge. The IG $\mathrm{LD}_{50}$ values were expressed in MIPLD ${ }_{50}$ units to facilitate comparison between different neurotoxin preparations.

\section{Results}

\subsection{Oral toxicity}

To compare the relative oral toxicities of multiple BoNT serotypes, a set of IG challenge experiments were performed in which $200 \mu \mathrm{L}$ of each neurotoxin was delivered into the stomachs of mice as described in methods, and the mice were monitored for mortality for 14 days. The IG $\mathrm{LD}_{50}$ for each neurotoxin preparation was expressed in the equivalent number of MIPLD ${ }_{50}$ units (Figure 1). There was an apparent trend in which the IG toxicity of the crude and progenitor forms was greater than the derivative form for a majority of the serotypes. This trend was most noticeable for the crude and progenitor 
forms of serotype A and serotype B, which were 2-3 orders of magnitude more toxic than the derivative form. Serotype $\mathrm{E}$ and serotype $\mathrm{F}$ also followed this trend in which the crude and progenitor preparations were more toxic than the derivative, but the magnitude of the difference was less with these neurotoxins. Minimal, if any, differences were noted between the crude, progenitor, and derivative preparations of serotype C. Crude and progenitor preparations of serotype B displayed the highest level of toxicity by the IG route, as they were at least 20 times more toxic than the other serotypes.

Figure 1 BoNT IG toxicity

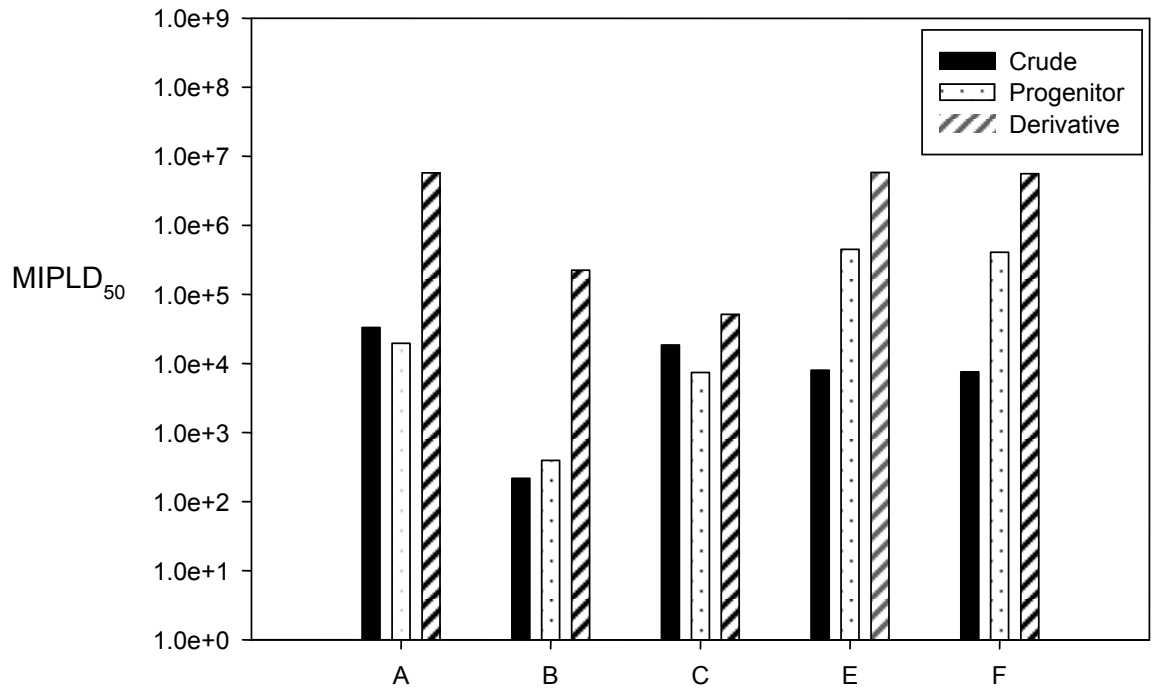

Notes: The BoNT serotype is shown on the $\mathrm{x}$-axis, and IG LD50 values expressed as MIPLD50 units are shown on the y-axis. Black bars correspond to crude, dotted bars correspond to progenitor, and upward diagonal line bars correspond to derivative.

\subsection{In vitro stability}

To gain insight into the impact of simulated GI tract conditions on the activity of the BoNTs, neurotoxin aliquots were exposed to $\mathrm{SGF} /$ pepsin and $\mathrm{SIF} /$ pancreatin at $35^{\circ} \mathrm{C}$, followed by evaluation of the remaining toxicity of each sample in the MPA. Exposures were conducted for 30 minutes, as this has been shown to be the amount of time required for binding and transcytosis of BoNT to occur in human gut epithelial cells (Ahsan et al., 2005). Percent activity remaining was calculated by dividing the MIPLD $D_{50} / \mathrm{mL}$ potency of each test sample by the MIPLD ${ }_{50} / \mathrm{mL}$ potency of controls not exposed to SGF/pepsin or $\mathrm{SIF} /$ pancreatin.

Exposure of the crude, progenitor, and derivative forms of the various BoNTs to SGF revealed an apparent trend in which the crude and progenitor forms were less susceptible to inactivation relative to the derivative form [Figure 2(a)]. Specifically, for serotypes A, $\mathrm{B}$, and $\mathrm{F}$, the crude form was least susceptible to SGF/pepsin, the progenitor form displayed an intermediate level of inactivation, and the derivative form showed the greatest degree of inactivation. This trend was less noticeable for serotypes $\mathrm{C}$ and $\mathrm{E}$. Notably, serotype $\mathrm{C}$ was an exception to this trend; stability in SGF and oral toxicity was nearly equivalent for each form of serotype $\mathrm{C}$. 
Figure 2 Effect of SGF and SIF on BoNT IG toxicity

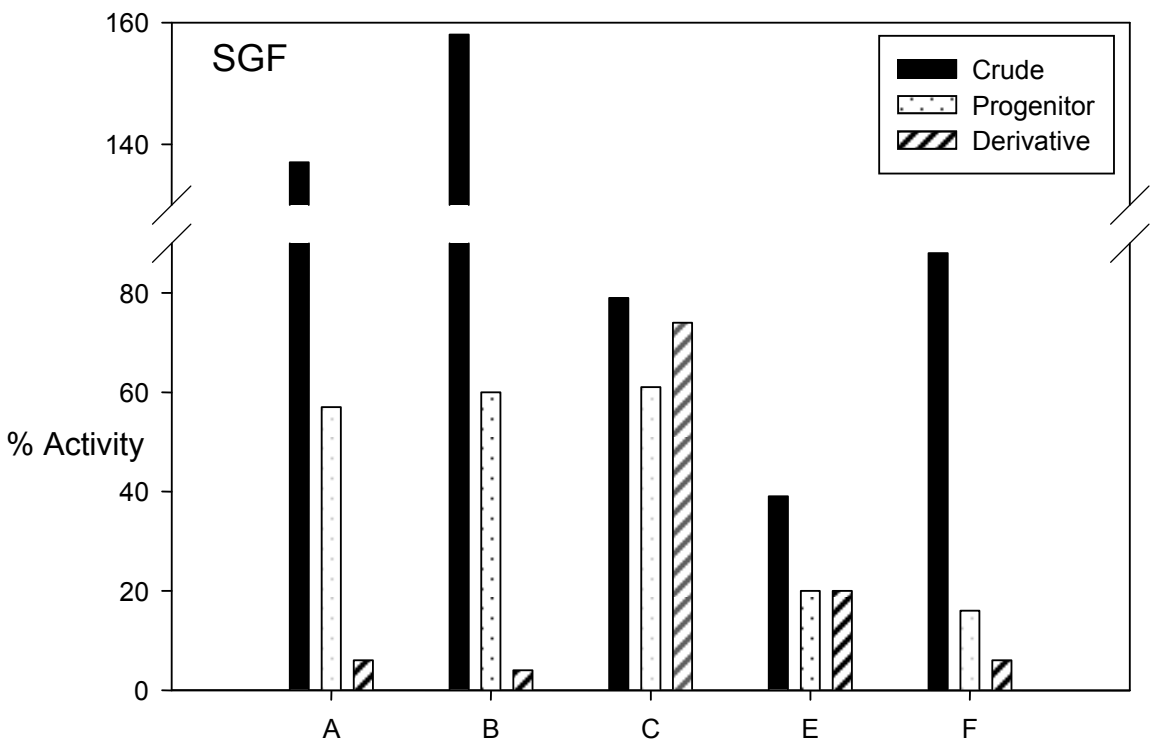

(a)

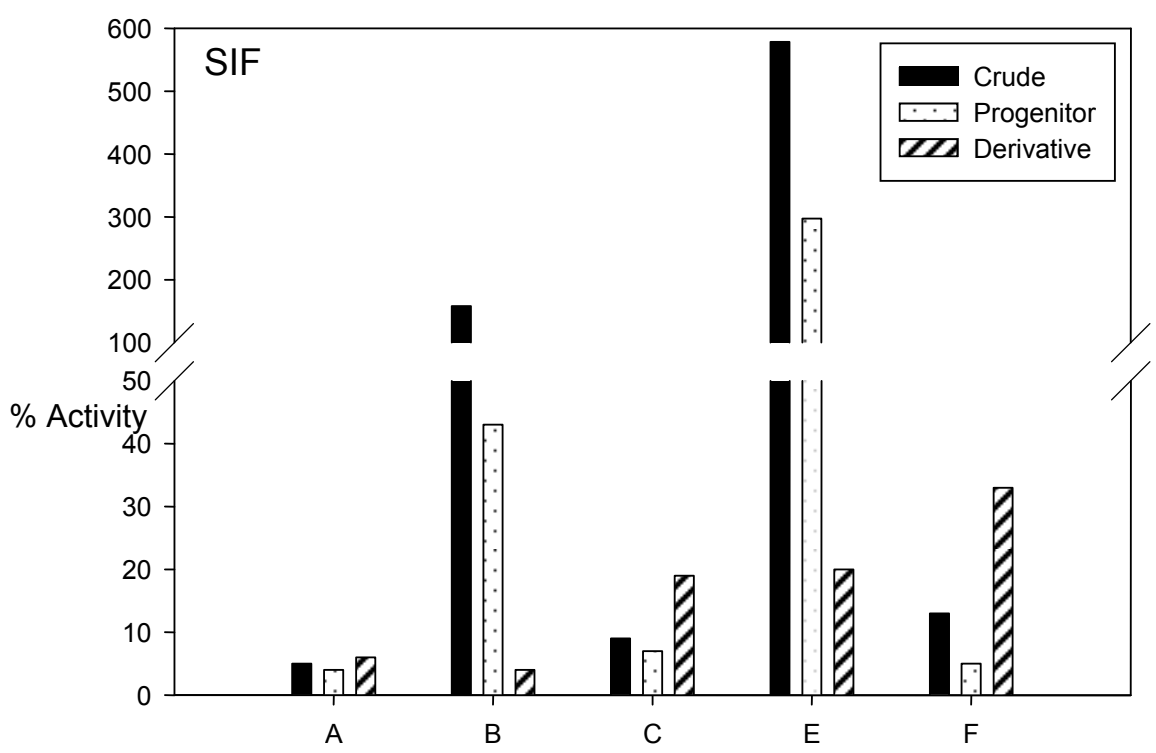

(b)

Notes: SGF and SIF results are shown in Figures 2(a) and 2(b), respectively. The BoNT serotype is shown on the $\mathrm{x}$-axis, and percent activity is shown on the $\mathrm{y}$-axis. Solid black bars correspond to crude, dotted bars correspond to progenitor, and upward diagonal line bars correspond to derivative. Percent activity was calculated by dividing the MIPLD $D_{50} / \mathrm{mL}$ potency of each BoNT test sample by the MIPLD ${ }_{50} / \mathrm{mL}$ potency of unexposed PBS controls. 
A majority of the BoNTs showed a different trend relative to the SGF/pepsin results following exposure of the crude, progenitor, and derivative forms to SIF/pancreatin [Figure 2(b)]. Specifically, the crude form of serotypes A, C, and F was inactivated to a greater degree by SIF/pancreatin as compared to SGF/pepsin. Additionally, for several serotypes, the crude and progenitor forms were inactivated to approximately equivalent or greater levels than the derivative forms. This suggests that the higher MW forms are more susceptible to intestinal fluids as compared to gastric fluids. Serotype B was a notable exception to this trend, suggesting that the high MW serotype B complexes remain intact at neutral $\mathrm{pH}$ values, thus protecting the neurotoxin against degradative enzymes. Additionally, activity of crude and progenitor serotype E and crude serotype B was enhanced by exposure to SIF/pancreatin. Collectively, these data suggest that stability in the gastrointestinal tract is an important factor in the oral toxicity of the BoNTs, and that the BoNTs are more stable in the stomach environment as compared to the intestinal tract.

\section{Discussion}

The observed higher IG toxicity and resistance to SGF/pepsin of the crude and progenitor preparations of the BoNTs relative to the derivative preparation is consistent with previously published studies (Kitamura et al., 1969; Ohishi and Sakaguchi, 1975, 1980; Sugii et al., 1977a, 1977b). It is thought that the non-toxic components that associate with the BoNTs to form larger MW complexes, specifically the HA and NTNH proteins (Inoue et al., 1996; Jin et al., 2009; Sakaguchi and Ohishi, 1984), protect the BoNTs against stomach acidity and digestive enzymes (Ohishi et al., 1977; Ohishi and Sakaguchi, 1980; Sakaguchi and Sakaguchi, 1974). Notably, serotype C did not follow this trend; the IG toxicity and resistance against SGF/pepsin of derivative serotype $\mathrm{C}$ was nearly equivalent to the crude and progenitor forms. This suggests that the serotype $\mathrm{C}$ neurotoxin may have unique structural properties that impart superior resistance to gastric conditions relative to the derivative form of other serotypes, and hence equivalent toxicity by the oral route relative to the higher MW forms. Additionally, a slight enhancement in activity was observed for crude serotypes A and B, suggesting that the treatment with SGF/pepsin minimally activated the two crude preparations.

In contrast to the SGF results, the type of BoNT preparation was less of a factor in stabilisation against SIF, suggesting that the higher MW forms were more susceptible to SIF than SGF. These observations may be explained by prior work suggesting that high MW complexes dissociate at approximately neutral $\mathrm{pH}$ conditions, but not under acidic conditions (Chen et al., 1998; Eisele et al., 2011; Fu et al., 1998). Dissociation of the complex in SIF/pancreatin ( $\mathrm{pH}$ 7.4) would expose the neurotoxin to various proteases that are part of the pancreatin enzyme mixture. Serotype B was a notable exception to this trend, suggesting that the high MW serotype B complexes remain intact at neutral $\mathrm{pH}$ values, thus protecting the neurotoxin against degradative enzymes. Enhanced stability in SIF/pancreatin may explain why serotype B displayed the highest level of oral toxicity out of the serotypes tested in this work [which correlates with previous observations of high serotype B oral toxicity (Ohishi et al., 1977)]. Additionally, activity of crude and progenitor serotype $\mathrm{E}$ and crude serotype $\mathrm{B}$ was enhanced by exposure to SIF/pancreatin. It may be possible that the in vitro trypsin treatment did not fully activate the serotype $\mathrm{E}$ preparations, and subsequent exposure to pancreatin resulted in completion of proteolytic 
activation. The slight enhancement in activity observed for crude serotype B likely indicates that a majority of the crude preparation was already activated prior to treatment with SIF/pancreatin. It should also be noted that enzymatic degradation in vivo following IP injection may also have contributed to the results observed in the SIF/pancreatin and $\mathrm{SGF} /$ pepsin stability experiments.

\section{Acknowledgements}

This publication was developed and funded under Agreement No. HSHQDC-07-C-00020 awarded by the US Department of Homeland Security for the management and operation of the National Biodefense Analysis and Countermeasures Center (NBACC), a Federally Funded Research and Development Center. The views and conclusions contained in this document are those of the authors and should not be interpreted as necessarily representing the official policies, either expressed or implied, of the US Department of Homeland Security. The Department of Homeland Security does not endorse any products or commercial services mentioned in this publication.

Battelle has an active Institutional Animal Care and Use Committee (IACUC) in compliance with PHS Policy, the Federal Animal Welfare Act, and Institute for Laboratory Animal Research guidelines. Battelle's Animal Welfare Assurance (number A3034-01) expires September 30, 2013. Protocols for all experiments using animals are reviewed and approved by Battelle's IACUC. The animal protocols used in this study were also approved by the National Biodefense Analysis and Countermeasures Center (NBACC) IACUC prior to initiation of experiments. The proper care and use of animals in the conduct of research described here is the responsibility of the Study Veterinarian, Study Director, and Battelle management. Animals at Battelle are cared for in accordance with the guidelines set forth in the Guide for the Care and Use of Laboratory Animals (1996, National Research Council) and in the regulations and standards as promulgated by the Agricultural Research Service, USDA, pursuant to the Animal Welfare Act of August 24, 1966, as amended.

\section{References}

Ahsan, C.R., Hajnóczky, G., Maksymowych, A.B. and Simpson, L.L. (2005) 'Visualization of binding and transcytosis of botulinum toxin by human intestinal epithelial cells', The Journal of Pharmacology and Experimental Therapeutics, Vol. 315, No. 3, pp.1028-1035.

Bonventre, P.F. (1979) 'Absorption of botulinal toxin from the gastrointestinal tract', Reviews of Infectious Diseases, Vol. 1, No. 4, pp.663-667.

Chang, A.C. and Gupta, R.K. (1996) 'Stabilization of tetanus toxoid in poly(DL-lactic-co-glycolic acid) microspheres for the controlled release of antigen', Journal of Pharmaceutical Sciences, Vol. 85, No. 2, pp.129-132.

Chen, F., Kuziemko, G.M. and Stevens, R.C. (1998) 'Biophysical characterization of the stability of the 150-kilodalton botulinum toxin, the nontoxic component, and the 900-kilodalton botulinum toxin complex species', Infection and Immunity, Vol. 66, No. 6, pp.2420-2425.

Eisele, K.H., Fink, K., Vey, M. and Taylor, H.V. (2011) 'Studies on the dissociation of botulinum neurotoxin type A complexes', Toxicon, Vol. 57, No. 4, pp.555-565.

Fu, F.N., Sharma, S.K. and Singh, B.R. (1998) 'A protease-resistant novel hemagglutinin purified from type A Clostridium botulinum', Journal of Protein Chemistry, Vol. 17, No. 1, pp.53-60. 
Fujinaga, Y. (2010) 'Interaction of botulinum toxin with the epithelial barrier', Journal of Biomedicine and Biotechnology, Article ID Number 974943, 9pp.

Fujinaga, Y., Inoue, K., Watanabe, S., Yokota, K., Hirai, Y., Nagamachi, E. and Oguma, K. (1997) 'The haemagglutinin of Clostridium botulinum type $\mathrm{C}$ progenitor toxin plays an essential role in binding of toxin to the epithelial cells of guinea pig small intestine, leading to the efficient absorption of the toxin', Microbiology, Vol. 143, No. 12, pp.3841-3847.

Inoue, K., Fujinaga, Y., Watanabe, T., Ohyama, T., Takeshi, K., Moriishi, K., Nakajima, H., Inoue, K. and Oguma, K. (1996) 'Molecular composition of Clostridium botulinum type A progenitor toxins', Infection and Immunity, Vol. 64, No. 5, pp.1589-1594.

Jin, Y., Takegahara, Y., Sugawara, Y., Matsumura, T. and Fujinaga, Y. (2009) 'Disruption of the epithelial barrier by botulinum haemagglutinin (HA) proteins - differences in cell tropism and the mechanism of action between HA proteins of types A or B, and HA proteins of type C', Microbiology, Vol. 155, No. 1, pp.35-45.

Kitamura, M., Sakaguchi, S. and Sakaguchi, G. (1969) 'Significance of 12 S toxin of Clostridium botulinum type E', Journal of Bacteriology, Vol. 98, No. 3, pp.1173-1178.

Kiyatkin, N., Maksymowych, A.B. and Simpson, L.L. (1997) 'Induction of an immune response by oral administration of recombinant botulinum toxin', Infection and Immunity, Vol. 65, No. 11, pp.4586-4591.

Maksymowych, A.B. and Simpson, L.L. (2004) 'Structural features of the botulinum neurotoxin molecule that govern binding and transcytosis across polarized human intestinal epithelial cells', The Journal of Pharmacology and Experimental Therapeutics, Vol. 310, No. 2, pp.633-641.

Maksymowych, A.B., Reinhard, M., Malizio, C.J., Goodnough, M.C., Johnson, E.A. and Simpson, L.L. (1999) 'Pure botulinum neurotoxin is absorbed from the stomach and small intestine and produces peripheral neuromuscular blockade', Infection and Immunity, Vol. 67, No. 9, pp.4708-4712.

Mao, Y., Doyle, M.P. and Chen, J. (2006) 'Role of colanic acid exopolysaccharide in the survival of enterohaemorrhagic Escherichia coli O157:H7 in simulated gastrointestinal fluids', Letters in Applied Microbiology, Vol. 42, No. 6, pp.642-647.

Ohishi, I. and Sakaguchi, G. (1975) 'Molecular construction of Clostridium botulinum type F progenitor toxin', Applied Microbiology, Vol. 29, No. 4, pp.444-447.

Ohishi, I. and Sakaguchi, G. (1980) 'Oral toxicities of Clostridium botulinum type C and D toxins of different molecular sizes', Infection and Immunity, Vol. 28, No. 2, pp.303-309.

Ohishi, I., Sugii, S. and Sakaguchi, G. (1977) 'Oral toxicities of Clostridium botulinum toxins in response to molecular size', Infection and Immunity, Vol. 16, No. 1, pp.107-109.

Sakaguchi, G. and Ohishi, I. (1984) 'Structure and function of botulinum toxins', in Alouf, J.E. et al. (Eds.): Bacterial Protein Toxins, pp.435-443, Academic Press, London, UK.

Sakaguchi, G. and Sakaguchi, S. (1974) 'Oral toxicities of Clostridium botulinum type E toxins of different forms', Japanese Journal of Medical Science \& Biology, Vol. 27, No. 4, pp.241-244.

Sugii, S., Ohishi, I. and Sakaguchi, G. (1977a) 'Correlation between oral toxicity and in vitro stability of Clostridium botulinum type A and B toxins of different molecular sizes', Infection and Immunity, Vol. 16, No. 3, pp.910-914.

Sugii, S., Ohishi, I. and Sakaguchi, G. (1977b) 'Intestinal absorption of botulinum toxins of different molecular sizes in rats', Infection and Immunity, Vol. 17, No. 3, pp.491-496.

Sugiyama, H., Dasgupta, B.R. and Yang, K.H. (1974) 'Toxicity of purified botulinal toxin fed to mice', Proceedings of the Society for Experimental Biology and Medicine, Vol. 147, pp.589-591. 